\title{
La inteligencia emocional: una herramienta necesaria para el liderazgo. Concepto y componentes
}

\author{
Armando Martin Martínez Percy ${ }^{1}$
}

\begin{abstract}
Resumen
La inteligencia emocional será la herramienta y/o recurso más importante del siglo XXI para sobrevivir a una época de "cambio permanente". Es básica para incrementar el rendimiento profesional y el bienestar personal especialmente en profesiones complejas, como las que incluyen las relaciones personales como centro y principal motor de la actividad: educación, recursos humanos, comunicación, etc. Daniel Goleman (1995), conocido como el autor de la inteligencia emocional, argumenta que las competencias humanas como auto-conciencia, auto-disciplina, persistencia y empatía, tienen más consecuencias en la vida de la persona que el cociente intelectual.
\end{abstract}

Goleman (1995) en sus investigaciones demuestra que la inteligencia emocional puede ser fomentada y fortalecida en todos nosotros, y cómo la falta de la misma puede influir en el intelecto o arruinar una carrera. Por todo esto, en este artículo de reflexión daremos cuenta acerca de la importancia de aprender sobre la inteligencia emocional, su concepto y sus componentes, la manera en que estos influyen en nuestro acontecer diario en los diferentes ambientes en que nos movemos, con el fin de aprender a desarrollar y canalizar nuestras emociones para obtener habilidades personales que proporcionen una diferencia sustancial en el contexto personal y profesional.

Palabras clave: autoconciencia, autorregulación, empatía, inteligencia emocional, habilidades, motivación.

1 Administrador de Empresas, Especialista en Gerencia del Recurso Humano, Diplomado en Docencia, Diplomado en Tutoría virtual de curso académico, tutor hora cátedra de Ecacen Cead Corozal, Universidad Nacional Abierta y a Distancia - UNAD. Grupo de investigación: Tendencias armando.martinez@unad.edu.co, Colombia. 


\title{
Emotional intelligence: a necessary tool for leadership. Concept and components
}

\begin{abstract}
The emotional intelligence (EI) will be the most important tool and/or resource of the XXI century to survive in a season of "constant change". EI is essential to increase the professional performance and the personal well-being especially in complex professions that include personal relations as center and principal motor of the activity: education, human resource, communication, and so on. Daniel Goleman (1995) is known as the author of EI, who claims that the human competences as selfawareness, self-discipline, persistence and empathy, have more impact in the person's life than the intelligence quotient (IQ).

Goleman (1995) shows in his reasearch that EI can be fomented and strengthened in everyone, and the lack of it can influence the intellect or ruin a career. Thus this think-piece will review the importance of learning about the EI, its concept and components, the way in that influence the daily happenings in the different environments., attempting to learn to develop and focus emotions getting personal skills that provide a substantial difference in personal and professional context.
\end{abstract}

Key words: Empathy, emotional intelligence, motivation, self-awareness, self-regulation, skills. 


\section{lntroducción}

Una persona puede tener un Coeficiente Intelectual (CI) elevado y una formación técnica o profesional impecable; pero ser incapaz de dirigir su propia vida o a un equipo hacia el éxito. Solo pueden ser líderes efectivos quienes tienen o aprenden la "Inteligencia Emocional" (IE), es decir la capacidad para captar las emociones propias y la de los demás y conducirlas hacia un resultado positivo. La IE es un campo de estudio que surgió en la década de los 90 como reacción al enfoque de inteligencias meramente cognitivas, sumando críticas a los detractores de los test de inteligencia tradicional.

Este enfoque se hizo rápidamente popular en la prensa no científica, entre otras razones porque emitía un mensaje novedoso y atractivo: se puede tener éxito en la vida sin tener grandes habilidades académicas. El libro divulgado de Daniel Goleman (1995) se convirtió rápidamente en el más vendido, aunque la investigación al respecto se encontraba aun en sus inicios. Goleman (1995) manifiesta que existen situaciones importantes que reclaman distintos tipos de liderazgo. Por ejemplo, la gran mayoría de las fusiones necesitan al frente un negociador sensible, mientras que los "revolcones" o situaciones difíciles requieren una autoridad mucho más enérgica. Es sabido que los estilos de líderes varían: unos son tímidos y analíticos, mientras otros proclaman a gritos sus ideas desde la cima. Sin embargo, se ha encontrado que los líderes más efectivos se parecen en un punto crucial: todos tienen un alto grado de lo que se ha llamado IE, no significa esto que el CI, las habilidades técnicas sean irrelevantes; pero como puntos de partida, constituyen requerimientos de entrada a las altas posiciones gerenciales.

Hoy en día las reglas del mundo laboral han cambiado, se requieren personas que tengan capacidad de manejarse tanto así mismas como a los demás; dichas reglas se presume no son aprendidas en la academia, por lo cual debemos identificar herramientas para el desarrollo de capacidades que permitan equiparnos de conocimientos en el manejo de nuestras cualidades personales como las que propone la IE (Goleman, 2003).

Recientes estudios e investigaciones, demuestran claramente que la IE es uno de los grandes componentes del liderazgo. Una persona que carezca de esta puede tener el mejor entrenamiento del mundo, una mente analítica e incisiva y un sin número de ideas, pero no será un gran líder en las actividades que desempeña o desarrolla a nivel personal y profesional.

Por todo lo anterior, el presente artículo de reflexión tendrá como objetivo principal proporcionar información sobre el concepto novedoso y los componentes de la IE, sus usos e importancia en el ámbito personal y profesional. De igual forma despejaremos los siguientes interrogantes: ¿Por 
qué algunas personas parecen dotadas de un don especial que les permite vivir bien, aunque no sean las que más se destacan por su inteligencia? ¿Por qué no siempre el alumno más inteligente termina siendo el más exitoso? ¿Por qué unos son más capaces que otros para enfrentar contratiempos, superar obstáculos y ver las dificultades bajo una óptica distinta? ¿De qué depende el éxito y que factores entran en juego cuando personas con un alto CI fracasan en sus actividades cotidianas, mientras que otras con un CI más modesto triunfan con más facilidad?

\section{Algunas ideas iniciales}

El artículo se propone presentar una mirada sobre la teoría de IE, básicamente tomando como referente central lo planteado por Daniel Goleman (1.995). Además, se tomaron como referentes investigaciones realizadas en personas y grupos de personas aplicando la teoría de la IE, documentos y vídeos. Lo anterior, con el objetivo de explicar la situación estudiada e interpretarla. De igual forma, se interpretó la información recogida de acuerdo a nuestros valores y controlando la influencia de ésta mediante la autoconciencia y examen riguroso de la misma. Por ello, con este artículo no se pretende demostrar teorías existentes, sino que se busca generar un proceso de sensibilización a partir del resultado final de este escrito.

\section{Interpretando la información}

La vida da muchas vueltas, nada permanece igual, todo cambia y en un mundo tan complejo como el nuestro, dominar las emociones empieza a ser un valor, tanto en los negocios, en la vida pública, en la familia, como en todos aquellos ambientes en que nos movemos. Por ello, el éxito de nuestras vidas desde cualquier punto de vista: personal, familiar, profesional, etc. depende del aprendizaje de la IE y la buena noticia es esta: que todos podemos aprender la IE, la cual nos enseña a controlar y administrar correctamente nuestras emociones, incrementando la autoconciencia, facilitando elegir aptitudes que nos hacen tolerar las presiones y frustraciones, mejorando nuestra capacidad de trabajar en equipo, crecer socialmente y aumentando nuestra motivación, lo cual nos otorga capacidad de liderazgo, brindándonos más posibilidades de éxito y de crecimiento personal.

Y para dar cuenta de lo contrario, podemos ver con ejemplos y estudios recientes de hace pocos años y publicados en revistas de muy alto nivel, que las personas con psicopatologías (depresión, esquizofrenia, trastornos agresivos, etc.) tienen en muchos casos déficit en sus habilidades, competencias emocionales y sociales. Dichos estudios psicopatológicos muestran por ejemplo, que los adolescentes con baja IE consumen más drogas legales como por ejemplo el tabaco y el alcohol. Estos estudios se han realizado en Estados Unidos y parte de Europa y dan como 
resultado que existe un factor de riesgo. Pero, no significa ello que los adolescentes con alta IE no consuman dichas drogas legales, sino que éstos son capaces de moderar su consumo y su conducta.

Estudios muestran también que incluso en las relaciones de parejas (satisfacción) influye la IE. Por ejemplo, se comparó entre tres tipos de parejas: parejas que entre él y ella tenían alta IE, parejas que sólo una de ellas tenía alta IE y parejas que ambos tenían baja IE. En dicho estudio se encontró que las parejas que tenían baja IE se diferenciaban de las otras parejas en que estaban insatisfechas, tenían muchos conflictos y rompían sus relaciones antes que las otras parejas.

Pero, en realidad en el caso que nos compete como el de la empresa, los ejecutivos y lideres saben que la motivación por ejemplo es más vital que las destrezas intelectuales o técnicas y es por ello que donde más se ha leído la IE, precisamente los libros de Daniel Goleman (1995), es aquí donde ha sido relevante, porque las grandes empresas u organizaciones no seleccionan a las personas por su hoja de vida y experiencia sino por sus habilidades emocionales y sociales, e incluso en las negociaciones; estudios de la universidad de Harvard muestran que las personas con más IE negocian mejor, pero sobre todo porque dejan más satisfechos a la otra persona con la que se negocia, y por su puesto la otra persona se va muy contenta y satisfecha.

En fin, para conocer y controlar las emociones propias y de extraños, se hace necesario desarrollar estas habilidades o componentes de la IE:

\section{Autoconocimiento}

2. Autocontrol

3. Auto motivación

\section{Empatía}

\section{Sociabilidad o habilidades interpersonales.}

Pero, que significa cada uno de estos componentes que sin duda son esenciales desarrollar:

1. Autoconocimiento: es el conocimiento de las propias emociones. Significa ser consciente de uno mismo: conocerse, conocer la propia existencia y ante todo el propio sentimiento de la vida. Es la capacidad de reconocer un sentimiento en el mismo momento en el que ocurre. El autoconocimiento se logra teniendo una actitud autocritica; haciendo una evaluación real, conociendo nuestros miedos, fortalezas y debilidades; nombrando o simbolizando nuestras emociones; teniendo confianza en uno mismo y reconociendo cómo los sentimientos nos afectan.

2. Autocontrol: capacidad para controlar nuestras emociones y sentimientos adecuándolos a los momentos. Es la capacidad de tranquilizarse y equilibrarse y de tener claro las consecuencias de ausencia. El autocontrol se 
logra cuando confiamos en nuestras decisiones; cuando conocemos nuestras responsabilidades; cuando nos liberamos de la ansiedad, respirando; cuando tenemos la capacidad de concentración y de prestar atención; cuando pensamos antes de hablar; cuando evitamos por ligereza hacer juicios erróneos.

3. Auto motivación: capacidad para motivarse uno mismo. Significa ser aplicado y juicioso para permanecer en la tarea, no dejándonos desalentar y ser capaces de ordenar las emociones al servicio de un objetivo esencial. La auto motivación se logra tomando la iniciativa para hacer las cosas e innovando; teniendo siempre una aptitud optimista, mente positiva $\mathrm{y}$ compromiso.

4. Empatía: es el reconocimiento de las emociones ajenas. Es la habilidad que nos permite concebir las carencias, emociones o dificultades de los demás, colocándose en su lugar o en sus zapatos para corresponder adecuadamente a sus reacciones emocionales; es decir, es la habilidad de conocer y entender lo que siente la otra persona. La empatía se logra escuchando a los demás, viendo las necesidades del otro, poniéndonos en situación del otro.

5. Sociabilidad o habilidades interpersonales: es el control de las relaciones; es la capacidad de conocer los sentimiento de otro y actuar de una manera, que se pueda dar nueva forma a esos sentimientos, siendo capaz de manejar las emociones del otro que es la esencia de mantener relaciones. Las habilidades sociales o interpersonales se logran teniendo en cuenta las normas de las buenas costumbres y conductas, siendo amables y saludando a las personas. Estando dispuesto a colaborar y a trabajar en equipo, manteniendo un alto grado de amistad y compromiso con los compañeros.

Se encuentra entonces, que diferentes escenarios proponen la enseñanza y el aprendizaje de la IE básica y necesaria para el éxito de nuestra vida. Y estos temas de aprendizaje y enseñanza incluyen por ejemplo: temas de conciencia de sí mismo:

1. En el sentido de reconocer los propios sentimientos y construir un vocabulario adecuado para expresarlos.

2. Aprender a ver los vínculos existentes entre pensamientos, sentimientos y reacciones.

3. Saber si los pensamientos o los sentimientos están gobernando las decisiones.

4. Ver las consecuencias posibles de elecciones alternativas.

5. Aplicar todas estas percepciones en decisiones sobre temas tales como drogas, tabaco y sexo.

Tomar conciencia del sí mismo también implica tomar conciencia de las propias fortalezas y debilidades, y verse a uno mismo bajo una luz optimista, pero realista, evitando así una baja en la propia autoestima. Otro punto que enfatiza 
es el de manejo de las emociones, y el darse cuenta de lo que hay detrás de cualquier sentimiento (el dolor que provoca enfado), para así aprender formas de manejar la ansiedad, el enojo y la tristeza. También se pone énfasis en hacerse cargo de las responsabilidades que generan los actos y las decisiones en asumir los compromisos.

Por otro lado, es importante destacar y tener claridad acerca de los usos de la IE como por ejemplo en el desarrollo personal de las personas, en la crianza y educación de los hijos, en el trabajo y en toda relación del ser humano. La IE es considerada como el más importante de los factores que intervienen en el ajuste personal, en el éxito en las relaciones personales y en el rendimiento en el trabajo. Con una IE desarrollada se pueden conseguir mejoras en el mundo laboral y en nuestras relaciones sociales, ya que la competencia emocional influye en todos los ámbitos importantes de la vida.

\section{Algunas ideas para la discusión}

¿Por qué algunas personas parecen dotadas de un don especial que les permite vivir bien, aunque no sean las que más se destacan por su inteligencia? ¿Por qué no siempre el alumno más inteligente termina siendo el más exitoso? ¿Por qué unos son más capaces que otros para enfrentar contratiempos, superar obstáculos y ver las dificultades bajo una óptica distinta? ¿De qué depende el éxito y qué factores entran en juego cuando personas con un alto C.I fracasan en sus actividades cotidianas, mientras que otras con un C.I más modesto triunfan con más facilidad?

La respuesta a estos interrogantes está en la emociones, y en la capacidad para entenderlas y manejarlas. La IE es parte de nuestra inteligencia global, una parte a menudo negada y desestimada, opacada por el brillo de la razón y del CI, más fácil de definir y medir. Para dar cuenta de ello, podemos analizar entre tantos ejemplos el siguiente: En un foro de negocios para directivos en los EE.UU, Goleman (1995) tuvo la oportunidad de hacer una encuesta informal sobre cuántos directivos se habían destacado del resto en la universidad, y quizás de doscientos directivos, había cuatro o cinco. Indudablemente no era mucho.

Esto según las investigaciones es porque el CI sólo predice entre el 4 y el 10 por ciento del éxito profesional. Esto deja afuera una contribución amplia de otros factores. Uno de ellos es la IE, las habilidades que distinguen a personas sobresalientes en cientos de organizaciones.

En el estudio el autor (Goleman, 1995) observó sólo dos cosas: ¿Cuántas de esas habilidades se basan en el CI y el conocimiento técnico, en habilidades puramente cognitivas y cuantas pertenecen al dominio de la IE? Resultó que para todo tipo 
de trabajos a la hora de diferenciar a las "estrellas" del resto, la I.E tiene el doble de importancia que las habilidades cognitivas. A mayor nivel en una organización, mayor es su importancia. Así que para los líderes de primer nivel en esos modelos de competencias el $80 \%$ y el $90 \%$ por ciento de las habilidades pertenecen al dominio de la IE. No es que el CI y el conocimiento técnico no importen sino que simplemente son habilidades básicas.

El CI es el indicador más fuerte de consecución de trabajo, que exigen para puede obtener y mantener una persona en un trabajo. Sin embargo, una vez que la persona está contratada no predice si será un trabajador sobresaliente. ¿Quiénes serán las estrellas, los líderes? Todo eso tiene que ver con la IE, es decir, con cómo nos manejamos y cuan efectivos somos en las relaciones personales.

La diferencia entonces, radica en lo que el autor (Goleman, 1995) llama IE. Lo expresa claramente en su libro, cómo la correcta administración de nuestras emociones puede fortalecernos e inclusive ayudar a mejorar carreras profesionales, y en cómo la falta de la misma puede influir en el intelecto de las personas.

\section{A manera de conclusiones}

El propósito de este artículo ha sido sensibilizar a los lectores sobre la importancia de la educación explicita de las emociones y de los beneficios personales y sociales que conlleva, puesto que la globalización de la economía ha desencadenado una fuerte competencia en el ámbito laboral. Como hemos comentado ya no bastan las habilidades clásicas que aprendemos durante la formación académica, las cuales están básicamente relacionadas con la práctica del conocimiento adquirido. Ahora es necesario incluir dentro de la formación ciertas habilidades personales, no ligadas a la academia, sino al crecimiento personal como seres humanos capaces de cambiar y adaptarnos a nuestros entornos, los cuales son cada vez más competidos.

El desarrollo de la IE dará a quienes deseen aprender acerca de esta nueva herramienta del liderazgo la oportunidad de evolucionar en conjunto con el ambiente económico, y así, la posibilidad de sobrevivir en esta nueva era en la que el manejo de los negocios, e incluso la permanencia en un empleo es cada vez más difícil. De igual forma, nos damos cuenta que no sólo se puede ser una persona exitosa únicamente dependiendo de nuestro intelecto, sino que el manejo correcto y adecuado de nuestras emociones es el pilar fundamental para llegar a nuestra o nuestras metas. 


\section{Referencias bibliográficas}

Álvarez Manilla, J. M., Valdés Krieg, E. \& Curiel de Valdés, A. B. (2006). Inteligencia emocional y desempeño escolar. Revista Panamericana de Pedagogía, 9, 9-33. APA, 2009, p.180 n1.

Covey S. R. (1995). Los siete hábitos de las personas altamente efectivas. México: Paidós.

Goleman, D. (1996). La Inteligencia Emocional. Buenos Aires: Javier Vergara.

Goleman, D. (2000). La inteligencia emocional: Por qué es más importante que el cociente intelectual. México: Ediciones $\mathrm{B}$.

Guía a la redacción en el estilo APA, 6ta edición. Biblioteca de la Universidad Metropolitana Sylvia Zavala Trías, MLS

http://es.wikipedia.org/wiki/Inteligencia_emocional

http://webs.uvigo.es/pmayobre/master/textos/evangelina_garcia/inteligencia_ emocional.pdf

http://www.cibem.org/paginas/img/apa6.pdf

http://www.inteligencia-emocional.org/trabajoyempresa/casos2.htm

http://www.resumido.com/es/libro.php/190

González, J.A., y Fernández, R. (2007) La empresa Emocionalmente Inteligente. Cluster Conocimiento.

Lane, H. y Beauchamp, M. (1985). Comprensión del Desarrollo Humano. México: Pax.

Reig Pintado, D. (1994). Reto al cambio. México: Mc Graw Hill.

Rodríguez E., M. y Márquez§ A., M. (1988). Manejo de problemas y toma de decisiones, México: Manual Moderno.

Steiner, C. (1997). La Educación Emocional. § Buenos Aires: Javier Vergara.

Tomado de: Badal Mauricio. Elaboración de referencias y citas según las normas de la American Psychological Association. [En línea] 1997. [Fecha de acceso 16 de Abril de 2012]. URL disponible en: http://www.monografias.com/apa.shtml

Uzcátegui, L. J. (1998). Emociones Inteligentes: El Manual de la Inteligencia Emocional. Caracas: LithoPolar.

Vallés, A. y Vallés, C. (2003). La autorregulación para el afrontamiento emocional. En Vallés, A. y Vallés, C. (2003). Psicopedagogía de la Inteligencia Emocional. Valencia: Promolibro. 


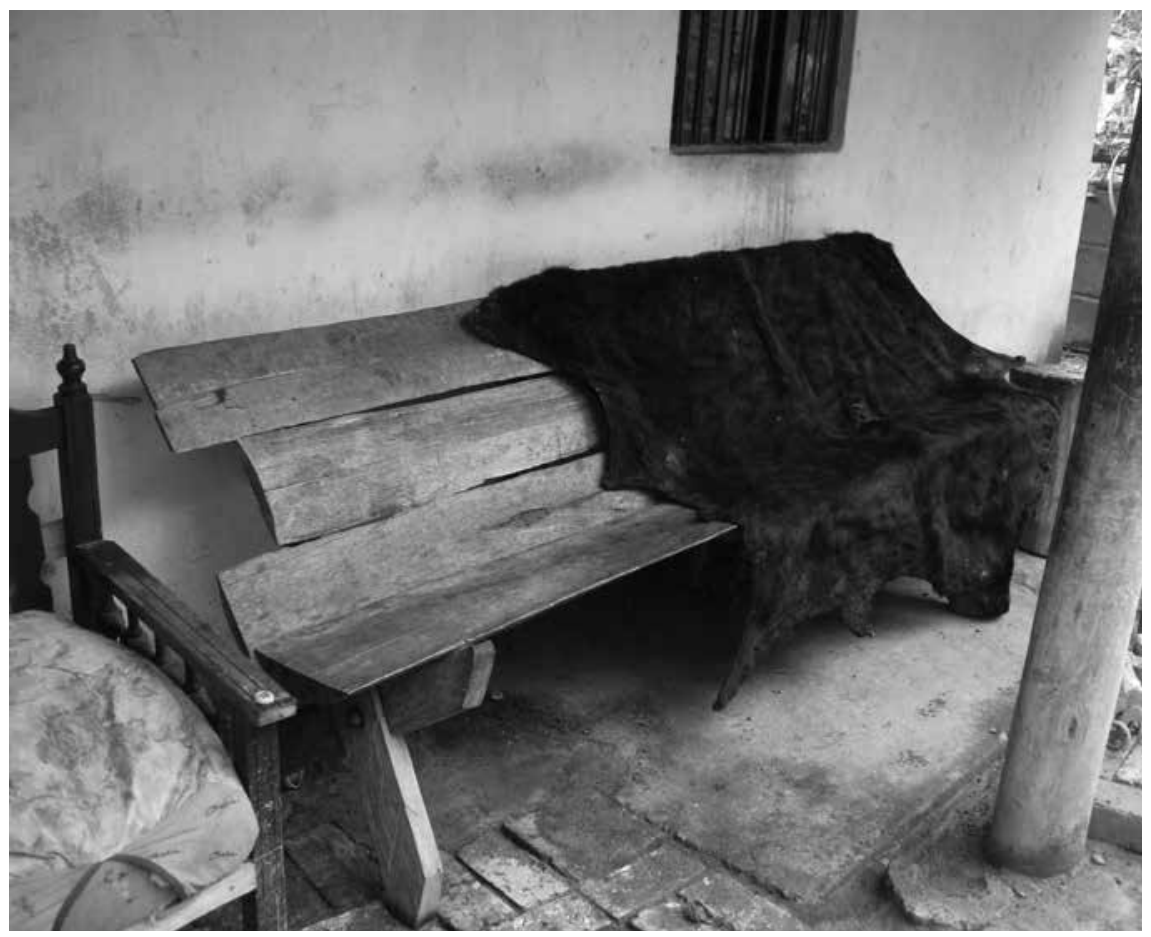

Fotografia: José Enrique Castillo 